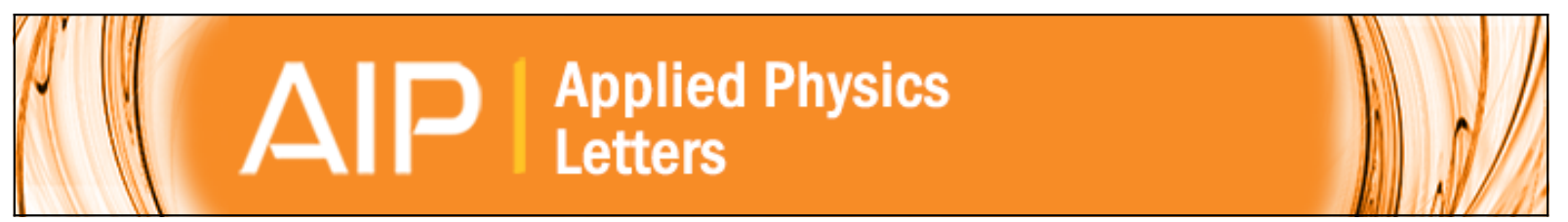

\title{
Emission and absorption cross-sections of an Er:GaN waveguide prepared with metal organic chemical vapor deposition
}

Q. Wang, R. Dahal, I.-W. Feng, J. Y. Lin, H. X. Jiang, and R. Hui

Citation: Applied Physics Letters 99, 121106 (2011); doi: 10.1063/1.3636418

View online: http://dx.doi.org/10.1063/1.3636418

View Table of Contents: http://scitation.aip.org/content/aip/journal/apl/99/12?ver=pdfcov

Published by the AIP Publishing

\section{Articles you may be interested in}

Excitation cross section of erbium-doped $\mathrm{GaN}$ waveguides under $980 \mathrm{~nm}$ optical pumping

Appl. Phys. Lett. 105, 051106 (2014); 10.1063/1.4892427

Violet-light spontaneous and stimulated emission from ultrathin In-rich InGaN/GaN multiple quantum wells grown by metalorganic chemical vapor deposition

Appl. Phys. Lett. 93, 161905 (2008); 10.1063/1.3002300

Optical properties of $\mathrm{n}$-doped Ga 1 - x Mn x N epitaxial layers grown by metal-organic chemical-vapor deposition in mid and far $(5-50 \mu \mathrm{m})$ IR range

J. Vac. Sci. Technol. B 26, 52 (2008); 10.1116/1.2819259

Optical and magnetic behavior of erbium-doped GaN epilayers grown by metal-organic chemical vapor deposition

Appl. Phys. Lett. 91, 054106 (2007); 10.1063/1.2767992

Excitation dynamics of the $1.54 \mu \mathrm{m}$ emission in Er doped GaN synthesized by metal organic chemical vapor deposition

Appl. Phys. Lett. 90, 051110 (2007); 10.1063/1.2450641

\section{MULTIPHYSICS SIMULATION}

\section{Modeling and App Design Stories}
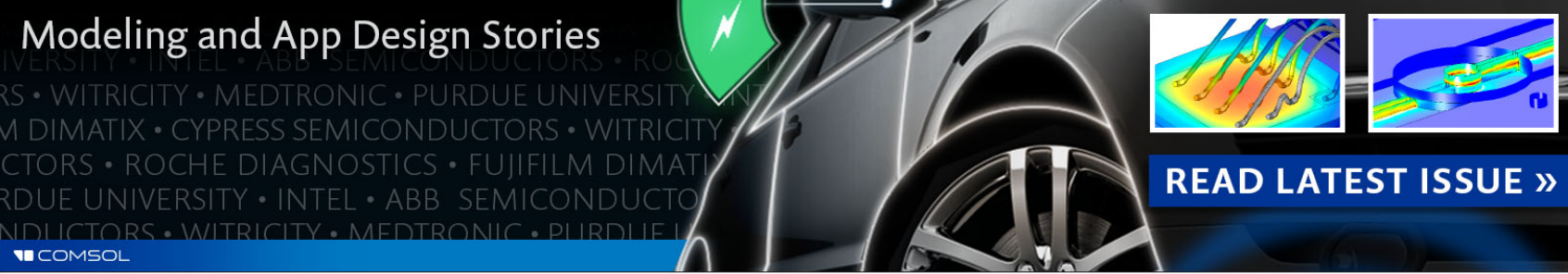

READ LATEST ISSUE ॥ 


\title{
Emission and absorption cross-sections of an Er:GaN waveguide prepared with metal organic chemical vapor deposition
}

\author{
Q. Wang, ${ }^{1}$ R. Dahal, ${ }^{2}$ I.-W. Feng, ${ }^{2}$ J. Y. Lin, ${ }^{2}$ H. X. Jiang, ${ }^{2}$ and R. Hui ${ }^{1, a)}$ \\ ${ }^{1}$ Department of Electrical Engineering and Computer Science, The University of Kansas, Lawrence, \\ Kansas 66045, USA \\ ${ }^{2}$ Department of Electrical and Computer Engineering, Texas Tech University, Lubbock, Texas 79409, USA
}

(Received 9 August 2011; accepted 19 August 2011; published online 20 September 2011)

\begin{abstract}
We repost the characterization of emission and absorption cross-sections in an erbium-doped GaN waveguide prepared by metal organic chemical vapor deposition. The emission cross-section was obtained with the Füchtbauer-Ladenburg equation based on the measured spontaneous emission and the radiative carrier lifetime. The absorption cross-section was derived from the emission cross-section through their relation provided from the McCumber's theory. The conversion efficiency from a $1480 \mathrm{~nm}$ pump to $1537 \mathrm{~nm}$ emission was measured, which reasonably agreed with the calculation based on the emission and absorption cross-sections. (C) 2011 American Institute of Physics. [doi:10.1063/1.3636418]
\end{abstract}

Erbium doped materials have attracted much attention for their operation in $1.5 \mu \mathrm{m}$ wavelength, and their application to optical communications. Doped into a solid host, $\mathrm{Er}^{3+}$ ion has allowable intra 4f shell transition from its first excited state $\left({ }^{4} \mathrm{I}_{13 / 2}\right)$ to the ground state $\left({ }^{4} \mathrm{I}_{15 / 2}\right)$, which corresponds to the wavelength of minimum optical loss in silica based optic fibers near $1.5 \mu \mathrm{m} .{ }^{1-4}$

III-nitride semiconductors are excellent host materials for Er ions due to their structural and thermal stabilities. ${ }^{5}$ It is well established that for wide band gap semiconductors such as $\mathrm{GaN}$, the Er related donor levels are deeper. Therefore, less electrons bound to Er related donor levels are thermally excited to the conduction band of $\mathrm{GaN}$ even at higher temperature, so there will be less thermal quenching compared to other semiconductor hosts. ${ }^{10}$

In the previous work, ${ }^{6}$ we have evaluated the carrier lifetime in the ${ }^{4} \mathrm{I}_{13 / 2}$ metastable energy band in room temperature. In addition, transition cross-sections are also fundamental parameters which determine the optical properties of lasers and amplifiers made of Er:GaN materials. In general, since it was difficult to directly measure both the absorption cross-section, $\sigma_{a}$, and the emission cross-section, $\sigma_{e}$, the typical procedure was to measure $\sigma_{a}$ first and then calculate $\sigma_{e}$ based on the measured results of $\sigma_{a}$ using the Einstein A and $\mathrm{B}$ coefficients for a two level system. ${ }^{7,8}$ In such an approach, the transition strength is sensitive to the Stark levels involved according to low temperature absorption and emission measurements. Therefore the above condition is not satisfied for Er:GaN at room temperature due to different populations of the sub-levels of each state. ${ }^{8}$

In contrast, the only requirement for the theory of McCumber is that the time necessary to establish a thermal distribution with each manifold should be short compared with the lifetime of that manifold. ${ }^{8,9}$ According to McCumber's theory, the absorption and emission cross sections can be related by

$$
\sigma_{e}(v)=\sigma_{a}(v) \exp [(h v-\varepsilon) / k T]
$$

a)Electronic mail: rhui@ku.edu. where $\varepsilon$ is the temperature-dependent excitation energy, which is the net energy needed to excite an $\mathrm{Er}^{3+}$ ion from the ${ }^{4} I_{15 / 2}$ to the ${ }^{4} I_{13 / 2}$ state at temperature $T$. Equation (1) indicates that frequency $v_{c} \equiv \varepsilon / h$ is the only intersection frequency between the two cross-section curves $\sigma_{a}$ and $\sigma_{e}$. $\sigma_{e}$ is smaller than $\sigma_{a}$ at frequencies higher than $v_{\mathrm{c}}$ and vice versa for $v<v_{\mathrm{c}}$. The excitation energy $\varepsilon$ can also be evaluated using an alternative definition

$$
N_{2} / N_{1}=\exp (-\varepsilon / k T),
$$

where $N_{2}$ and $N_{1}$ represent thermal equilibrium populations of the 1st excited state and the ground state, respectively, without any pump. If the accurate information for all the state's Stark components is known, $N_{2} / N_{1}$ can be evaluated by Eq. (3)

$$
\frac{N_{2}}{N_{1}}=\exp \left(\frac{-\Delta E_{21}}{k T}\right) \frac{1+\sum_{i=2}^{d_{2}} \exp \left(-\Delta E_{2 i} / k T\right)}{1+\sum_{i=2}^{d_{1}} \exp \left(-\Delta E_{1 i} / k T\right)},
$$

where $\Delta E_{21}$ represents the separation between the lowest sub-level of the 1st excited state and the lowest sub-level of the ground state, $\delta E_{2 i}$ is the energy difference between the $i$ th and the lowest sub-level of the 1 st excited state, while $\delta E_{1 i}$ is the energy difference between the $i$ th and the lowest sub-level of the ground state. With a proper assumption to simplify the electron structure, $\varepsilon$ can be determined by substituting Eq. (3) into Eq. (2), and $\sigma_{a}$ is related to $\sigma_{e}$ by the McCumber theory as shown in Eq. (1).

In fact, if the radiative carrier lifetime can be precisely measured, one can evaluate the emission cross-section through the Füchtbauer-Ladenburg (FL) equation,

$$
1 / \tau_{e}=8 \pi n^{2} c \int\left[\sigma_{e}(\lambda) / \lambda^{4}\right] d \lambda
$$

where $\tau_{e}$ is the radiative carrier lifetime of the 1 st excited state, $n$ is the refractive index of the solid host, $c$ is the light velocity in vacuum, and $\sigma_{\mathrm{e}}$ denotes the wavelength-dependent emission cross section. Within a narrow frequency band, the 
emission cross section in the FL equation is proportional to the spontaneous emission intensity $I(\lambda)$ which can be directly measured, and therefore Eq. (4) can be rewritten as

$$
\sigma_{e}(\lambda)=\frac{\bar{\lambda}^{4}}{8 \pi c n^{2} \tau_{e}} \frac{I(\lambda)}{\int I(\lambda) d \lambda},
$$

where $\bar{\lambda}$ is the average wavelength. Equation (5) shows that the emission cross section can be obtained from the carrier lifetime and the normalized emission spectrum, while the absolute power density values of the spontaneous emission are not necessary.

In the experiment, the waveguide is about $5 \mu \mathrm{m}$ wide and $2.6 \mathrm{~mm}$ long, which was prepared with metal organic chemical vapor deposition (MOCVD) as reported in Ref. 6 . A numerical calculation indicates that the overlap between the fundamental optical mode and the Er-doped layer is $\Gamma=19.8 \%$, which is commonly refereed to as the field confinement factor.

The experimental setup is shown in Figure 1. A $980 \mathrm{~nm}$ laser diode with single-mode fiber pigtail was used as the pump. The pump went through two 980/1550 nm wavelength division multiplexing (WDM) couplers to cutoff the spontaneous emission from the laser source in the $1550 \mathrm{~nm}$ wavelength window. The filtered pump was then guided and focused onto the end surface of the Er:GaN waveguide sample by a single mode fiber with a tapered tip. The same fiber was also used to collect the spontaneous emission generated from the waveguide. The spectral density of the emission $I(\lambda)$ was guided through the WDM coupler and detected by an optical spectrum analyzer (OSA1). In addition, this emission also passes through an optical bandpass filter with $1537 \mathrm{~nm}$ central wavelength and $1 \mathrm{~nm}$ optical bandwidth, and detected by a photodetector. The temporal waveform was then displayed by an oscilloscope, which allowed the measure of the time-domain modulation response of the spontaneous emission when the pump power was modulated. The carrier lifetime $\tau$ of the 1 st excited state of $\mathrm{Er}^{3+}$ can be evaluated in this process. All the measurements were made at room temperature.

Fig. 2 shows the normalized spontaneous emission spectral density measured by the OSA1. It indicates that the peak emission is at $1537 \mathrm{~nm}$ with the $13 \mathrm{~dB}(5 \%)$ bandwidth extending from $1470 \mathrm{~nm}$ to $1600 \mathrm{~nm}$. Following the theory outlined in Ref. 8, the band structure of $\left({ }^{4} \mathrm{I}_{13 / 2}\right)$ and $\left({ }^{4} \mathrm{I}_{15 / 2}\right)$ levels of $\mathrm{Er}^{3+}$ ions in $\mathrm{GaN}$ can be illustrated in the inset (a) of Fig. 2. In this diagram, the ground state and the 1 st excited state are split into eight and seven equally spaced sub-levels, respectively, due to the Stark effect. The transition between the lowest sub-level of the 1st excited state and the highest

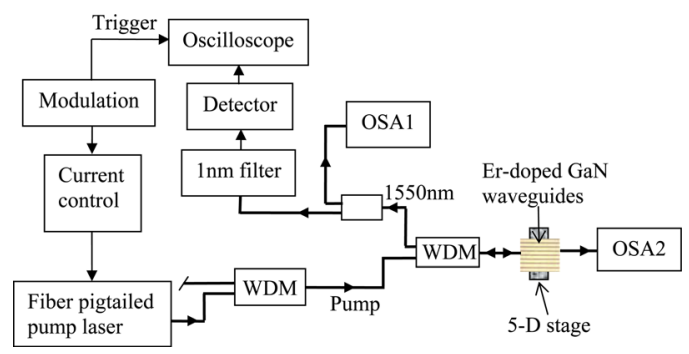

FIG. 1. (Color online) Experimental setup.

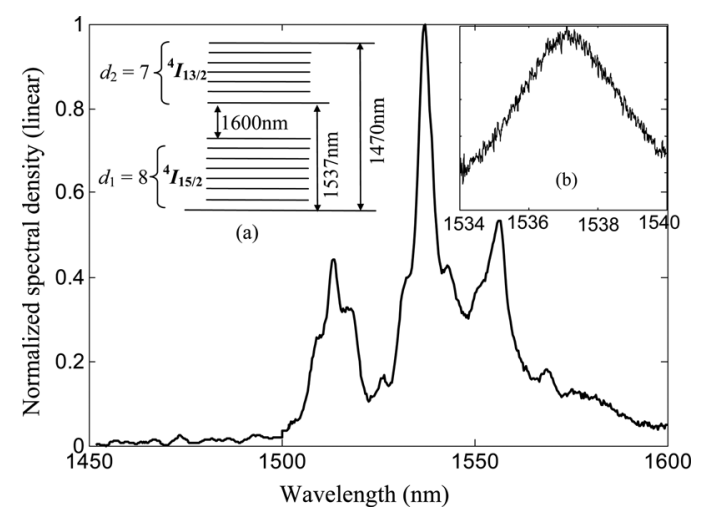

FIG. 2. Normalized emission spectrum of a Er:GaN waveguide measured by an OSA with $2 \mathrm{~nm}$ resolution. Inset (a): detailed band diagram of erbium ions in the 1st excited band and the ground state. Inset (b): detailed emission spectrum measured near $1537 \mathrm{~nm}$ with $0.5 \mathrm{~nm}$ OSA spectral resolution.

sub-level of the ground state produces $1600 \mathrm{~nm}$ emission wavelength, while the transition from the top of 1st excited state to the bottom of the ground state represents the $1470 \mathrm{~nm}$ emission. In addition, we assume that $1537 \mathrm{~nm}$ peak emission wavelength corresponds to the transition between the bottom of the 1st excited state and the bottom of the ground state.

With the above assumptions of energy band structure, the spacing between two adjacent sub-levels within each energy band can be easily calculated by, $7 \Delta E_{1}$ $=h c\left(\lambda_{2}^{-1}-\lambda_{4}^{-1}\right), 6 \Delta E_{2}=h c\left(\lambda_{1}^{-1}-\lambda_{2}^{-1}\right)$ with $\lambda_{1}=1470 \mathrm{~nm}$, $\lambda_{2}=1537 \mathrm{~nm}$, and $\lambda_{3}=1600 \mathrm{~nm}$.

Based on this simplified electronic structure, the excitation energy $\varepsilon$ defined by Eq. (2) can be obtained by combining Eqs. (3) and (4),

$$
\begin{aligned}
\varepsilon= & k T \ln \left[\frac{1-\exp \left(-\Delta E_{1} / k T\right)}{1-\exp \left(-\Delta E_{2} / k T\right)}\right]\left[\frac{1-\exp \left(-d_{2} \Delta E_{2} / k T\right)}{1-\exp \left(-d_{1} \Delta E_{1} / k T\right)}\right] \\
& -\Delta E_{21} .
\end{aligned}
$$

With the measured spontaneous emission spectrum shown in Fig. 2, the absolute values of $\sigma_{e}$ can be obtained by using Eq. (5) with the knowledge of the radiative carrier lifetime $\tau_{\mathrm{e}}$. Then $\sigma_{a}$ can be derived from this $\sigma_{e}$ using the McCumber's relation of Eq. (1) and the excitation energy $\varepsilon$ calculated from Eq. (6). Specifically for the band structure of Er:GaN discussed so far, $\varepsilon=6515.2 \mathrm{~cm}^{-1}$. Note that the measured carrier lifetime $\tau=\left(1 / \tau_{e}+1 / \tau_{a}\right)^{-1}$ consists of a radiative component $\tau_{e}$ and a non-radiative component $\tau_{a}$. Our previous measurement indicated two time constants, 1.5

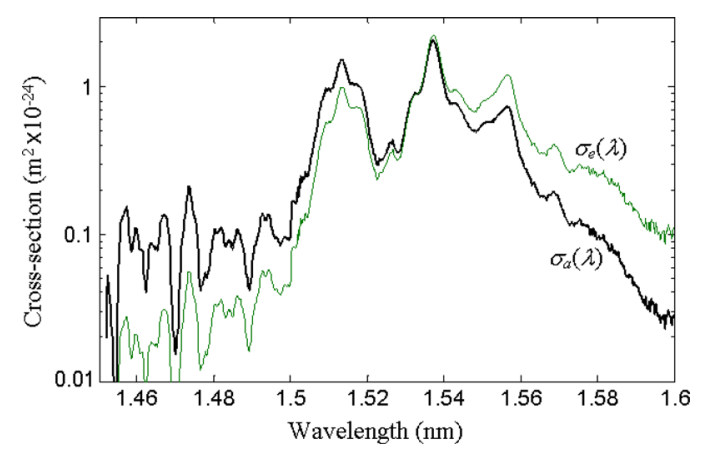

FIG. 3. (Color online) Absorption cross section $\sigma_{a}(\lambda)$ and emission cross section $\sigma_{e}(\lambda)$ for the ${ }^{4} \mathrm{I}_{13 / 2}$ to ${ }^{4} \mathrm{I}_{15 / 2}$ transition in an Er:GaN waveguide. 


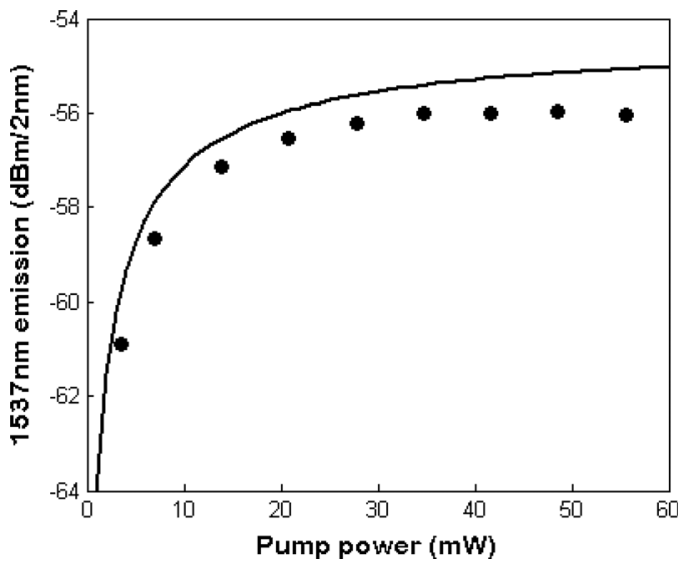

FIG. 4. Calculated (continuous line) and measured (circles) spontaneous emission power spectral density at $1537 \mathrm{~nm}$ wavelength as the function of the injected $1480 \mathrm{~nm}$ pump power.

ms and $2.8 \mathrm{~ms}$ for carrier decay in the Er:NaG waveguide. ${ }^{6}$ The shorter time constant involved non-radiative recombination and carrier up-conversion, while the longer time constant is responsible for the radiative recombination, so that we use $\tau_{e}=2.8 \mathrm{~ms}$ in the calculation of emission crosssection. Fig. 3 shows both absorption and emission cross sections determined using the above technique.

Although the absolute cross-section values of Er:GaN are on the same order of magnitude with those in erbiumdoped glasses, ${ }^{11}$ their cross-section spectral shapes are noticeably different. The emission and absorption crosssections shown in Fig. 3 exhibit a number of characteristic peaks due to the regular crystal lattice structure in the $\mathrm{GaN}$ material in which the erbium is embedded in. Note that in a recent measurement of Er:GaN material at a very low temperature of $T=10 \mathrm{~K},{ }^{12}$ the main emission peak at $1537 \mathrm{~nm}$ consists of 4 isolated emission lines with approximately 1 $\mathrm{nm}(\sim 0.0005 \mathrm{eV})$ wavelength separation between each other. However, in our measurement at room temperature, this spectral splitting was not observed. The inset (b) in Fig. 2 shows the emission peak at $1537 \mathrm{~nm}$ measured with a $0.5 \mathrm{~nm}$ spectral resolution, which clearly indicates a continuous emission spectrum. This line broadening is partly attributed to thermally induced lattice vibration of the semiconductor material. In addition, in the waveguide configuration investigated here, non-uniformity of crystal structure along the longitudinal direction of the waveguide may also contribute to this line broadening.

Since our measurement extends from $1450 \mathrm{~nm}$ to 1600 $\mathrm{nm}$, the efficiency of $1480 \mathrm{~nm}$ pumping can be evaluated. In this experiment, the power spectral density of spontaneous emission at $1537 \mathrm{~nm}$ was measured as the function of the injected $1480 \mathrm{~nm}$ pump power, as shown in Fig. 4. In order to test the accuracy of the cross-section values obtained so far, we also calculated the power spectral density of spontaneous emission at $1537 \mathrm{~nm}$ versus the pump power at $1480 \mathrm{~nm}$ wavelength based on the following relation, 1,13

$\rho_{A S E}=2 \Gamma \sigma_{e}\left(\lambda_{s}\right) h v_{s} \int_{0}^{L} \frac{N_{T} P_{p} e^{-2 \alpha z} \sigma_{a}\left(\lambda_{p}\right)}{P_{p} e^{-\alpha z}\left[\sigma_{a}\left(\lambda_{p}\right)+\sigma_{e}\left(\lambda_{p}\right)\right]+h v_{p} A / \tau} d z$, where $N_{\mathrm{T}}$ is the doping density of erbium ions. $\lambda_{p}$ is the pump wavelength, $\tau$ is the carrier lifetime, $\Gamma$ is the field confinement factor, $L$ is the length of the waveguide, $h v_{\mathrm{s}}$ is the signal photon energy, $A$ is the waveguide cross section, $\alpha$ is the attenuation, and $P_{p}$ is the pump optical power. According to the cross section values as shown in Fig. 3, the absorption and emission cross-sections at the $1480 \mathrm{~nm}$ pump wavelength are $\sigma_{a}\left(\lambda_{p}\right)=10^{-21} \mathrm{~cm}^{-2}, \sigma_{e}\left(\lambda_{p}\right)=3.2 \times 10^{-22} \mathrm{~cm}^{-2}$, and the emission cross-section at the $1537 \mathrm{~nm}$ signal wavelength is $\sigma_{e}\left(\lambda_{s}\right)=2.2 \times 10^{-20} \mathrm{~cm}^{-2}$. The calculated results shown as the solid line in Fig. 4 used the following parameters of the waveguide: $N_{T}=2 \times 10^{20} \mathrm{~cm}^{3}, \quad \Gamma=0.198$, $A=9.5 \times 10^{-8} \mathrm{~cm}^{3}, \tau=1.5 \mathrm{~ms}, L=2.6 \mathrm{~mm}$, and $\alpha=-1.5$ $\mathrm{dB} / \mathrm{mm}$.

With $50 \mathrm{~mW}$ pump power, the theoretical power spectral density emitted from the waveguide is $-55 \mathrm{dBm} / 2 \mathrm{~nm}$, which is about $1 \mathrm{~dB}$ (or 20\%) higher than the measured values shown as the open circles in the figure. This reduced emission efficiency is attributed to the non-radiative recombination and Auger recombination for the transition from the metastable level $\left({ }^{4} \mathrm{I}_{13 / 2}\right)$ to the ground level $\left({ }^{4} \mathrm{I}_{15 / 2}\right)$. Overall, the calculated results in Fig. 4 agree reasonably well with the measurement.

In conclusion, we have studied the optical properties of Erbium doped $\mathrm{GaN}$ and used a convenient procedure to determine the emission and absorption cross-sections in an Er:GaN waveguide sample prepared by MOCVD. The emission cross-section was obtained with the FüchtbauerLadenburg equation based on the measured spontaneous emission spectrum and the radiative carrier lifetime. The absorption cross-section was then derived from the emission cross-section through their relation provided from the McCumber's theory. Because of the crystal lattice structure in the semiconductor matrix, relatively narrow spectral lines exist in cross-section spectra of Er:GaN waveguides.

This work is supported by NSF (Grant No. ECCS0854619). Jiang and Lin gratefully acknowledge the support of Ed and Linda Whitacre Endowed Chair positions through the AT \& T Foundation.

${ }^{1}$ E. Desurvire, Erbium-doped Fibre Amplifiers: Principles and Applications (John Wiley \& Sons, Hoboken NJ, 1994).

${ }^{2}$ R. J. Mears, L. Reekie, I. M. Jauncey, and D. N. Payne, Electron. Lett. 23, 1026 (1987)

${ }^{3}$ P. C. Becker, N. A. Olsson, and J. R. Simpson, Erbium-Doped Fibre Amplifiers: Fundamentals and Technology (Academic, San Diego, CA, 1999).

${ }^{4}$ M. J. Connelly, Semiconductor Optical Amplifiers (Kluwer Academic, Dordrecht, The Netherlands, 2002).

${ }^{5}$ C. Ugolini, N. Nepal, J. Y. Lin, H. X. Jiang, and J. M. Zavada, Appl. Phys. Lett. 90, 051110 (2007).

${ }^{6}$ Q. Wang, R. Hui, R. Dahal, J. Y. Lin, and H. X. Jiang, Appl. Phys. Lett. 97, 241105 (2010).

${ }^{7}$ D. E. McCumber, Phys. Rev. 134, A299 (1964).

${ }^{8}$ W. J. Miniscalco and R. S. Quimby, Opt. Lett. 16, 258 (1991).

${ }^{9}$ J. A. Lázaro, J. A. Valles, and M. A. Rebolledo, Pure Appl. Opt. 7, 1363 (1998).

${ }^{10}$ R. Dahal, J. Y. Lin, H. X. Jiang, and J. M. Zavada, Top. Appl. Phys. 124, 115 (2010).

${ }^{11}$ W. J. Miniscalco, IEEE J. Lightwave Technol. 9, 234 (1991).

${ }^{12}$ N. Woodward, V. Dierolf, J. Y. Lin, H. X. Jiang, and J. M. Zavada, Opt. Mater. 33, 1059 (2011)

${ }^{13}$ C. Y. Chen, S. Wen, and S. Chi, Electron. Lett. 30, 889 (1994). 